\title{
ИССЛЕДОВАНИЕ КАЧЕСТВА ЖИЗНИ И УРОВНЯ ДЕПРЕССИИ У ПАЦИЕНТОВ С ХРОНИЧЕСКОЙ БОЛЕЗНЬЮ ПОЧЕК НА ПРОГРАММНОМ ГЕМОДИАЛИЗЕ
}

\section{TUDY OF QUALITY OF LIFE AND LEVEL OF DEPRESSION IN PATIENTS WITH CHRONIC KIDNEY DISEASE UNDER PROGRAM HEMODIALYSIS}

\section{A. Kardangusheva I. Almova A. Budnik A. Pshukova D. Urusbieva K. Pshukov}

Summary. The article examines the prevalence of depression in patients with chronic kidney disease (CKD) C5 receiving programmed hemodialysis (PGD) therapy and its impact on quality of life (Q0L). In 2018, 86 patients (60 men and 26 women) with CKD C5 receiving PGD treatment were examined at the North Caucasus Nephrology Center LLC. The average age of patients was $55.5 \pm 13.9$ years. The level of depression on the CES-D scale, the level of QOL-according to the KDQOL-SF ${ }^{\mathrm{TM}}$ questionnaire. The population of CKD C5 patients receiving PGD therapy examined by the authors is characterized by a high prevalence of depression. Symptoms of depression negatively affect the QOL of patients with CKD C5 on PGD. The introduction of the methods used to assess $Q 0 \mathrm{~L}$ and depression in the protocol of management of patients with CKD C5 in dialysis centers will allow monitoring the indicators of $\mathrm{QO} \mathrm{L}$ and symptoms of depression in patients for timely correction of their treatment. The results obtained by us can be used in planning psychological and psychopharmacological support of patients on PGD.

Keywords: chronic kidney disease, quality of life, depression, program hemodialysis.
Кардангушева Аксана Мухамедовна

Д.м.н., дочент, Кабардино-Балкарский государственный университет им. Х. М. Бербекова,

Нальчик

kardangush@mail.ru

Альмова Ирина Хаджиисмаиловна

К.м.н., дочент, Кабардино-Балкарский государственный университет им. Х. М. Бербекова,

Нальчик

almovairina@mail.ru

Будник Антонина Францевна

К.м.н., доцент, Кабардино-Балкарский государственный университет им. Х. М. Бербекова,

Нальчик

budnik74@mail.ru

Пиукова Альбина Анатольевна

Доцент, Кабардино-Балкарский государственный университет им. Х. М. Бербекова, Нальчик albina.75.75@bk.ru

урусбиева Джаннета Магометовна К.м.н., доцент, Кабардино-Балкарский государственный университет им. Х. М. Бербекова,

Нальчик

j_urusbieva1@mail.ru

Пиуков Каземир Русланович

Кабардино-Балкарский государственный университет им. Х. М. Бербекова, Нальчик kazik.pshukov@bk.ru

Аннотация. В статье изучается распространенность депрессии у пациентов с хронической болезнью почек (ХБП) С5, получающих терапию программным гемодиализом (ПГД) и его влияние на качество жизни (КЖ). На базе 000 «Северо-Кавказский Нефрологический Центр» в 2018 г. обследованы 86 пациентов (60 мужчин и 26 женщин) с ХБП С5, получающих лечение ПГД. Средний возраст пациентов составил 55,5 $\pm 13,9$ лет. Уровень депрессии по шкале CES-D, уровень KЖ - по опроснику KDQOL-SF ${ }^{\mathrm{T}}$. Обследованная авторами популяция пациентов ХБП С5, получающих терапию ПГД, характеризуется высокой распространенностью депрессии. Симптомы депрессии негативно воздействуют на КЖ пациентов с ХБП С5 на ПГД. Внедрение использованных нами методик оценки КЖ и уровня депрессии в протокол ведения пациентов с ХБП С5 в диализных центрах позволит мониторить показатели КЖ и симптомов депрессии пациентов для своевременной коррекции их лечения. Полученные нами результаты можно использовать при планировании психологического и психофармакологического сопровождения пациентов на ПГД.

Ключевые слова: хроническая болезнь почек, качество жизни, депрессия, программный гемодиализ. 


\section{Введение}

$\longrightarrow$ овершенствование тактики ведения пациентов с хронической болезнью почек (ХБП) С5, в частности улучшение технологии заместительной почечной терапии, позволило увеличить продолжительность жизни пациентов и обусловило актуальность улучшения ее качественного содержания. Вместе с тем, особенности диализной терапии, а именно длительность лечения, зависимость пациента от медицинской аппаратуры и персонала, продолжительность сеансов гемодиализа, ограничения, касающиеся свободы передвижения, питания, питьевого режима, инвалидизация, потеря работы с последующим ограничением круга общения, изменение собственной внешности, прием лекарственных препаратов, возникновение половой дисфункции сказываются на психологическом состоянии пациентов, приводя к снижению качества жизни (КЖ) и развитию депрессивной симптоматики [1]. Известно, что популяция пациентов с терминальной почечной недостаточностью, получающая заместительную почечную терапию, характеризуется низкими показателями КЖ и высокой распространенностью депрессии [2, 3]. По данным Kimmel P.L., Cukor D., Cohen S.D. (2007), депрессия у пациентов с ХБП C5 диагностируется в 10-60\% случаев, что значительно преВышает распространенность депрессии среди популяции и негативно влияет на течение и исход коморбидной патологии, в частности наиболее распространенных в диализной популяции сердечно-сосудистых заболеваний $[4,5]$. По данным различных исследователей, выраженность депрессии у пациентов, получающих заместительную почечную терапию, тесно связана с выживаемостью $[6,7]$.

Качество жизни представляет собой интегральную характеристику физической, эмоциональной и социальной активности пациента, основанную на его субъективном восприятии [8]. В ряде исследований установлены тесные корреляции между КЖ и выживаемостью пациентов на гемодиализе $[9,10]$. Изучение КЖ пациентов в повседневной практике позволяет с большей точностью определять меры их социальной и медицинской реабилитации. По мере совершенствования методов медицинской реабилитации расширяются возможности трудовой реабилитации и повышается КЖ пациентов.

Несмотря на то, что актуальность исследования различных аспектов КЖ и уровня депрессии у пациентов с терминальной почечной недостаточностью в зависимости от вида терапии, данная проблема требует дальнейшей разработки.

\section{Цель исслеАования}

Изучить распространенность депрессии у пациентов с ХБП С5, получающих терапию программным гемодиализом (ПГД) и его влияние на КЖ.

\section{Материалы и метолы}

В 2018 г. на базе ООО «Северо-Кавказский Нефрологический Центр» мы обследовали 86 пациентов (60 мужчин и 26 женщин) с ХБП С5, получающих лечение ПГД.

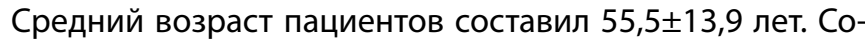
циально-демографическая характеристика пациентов обследованных пациентов представлена в табл. 1. Обращает на себя внимание преобладание среди обследованных нами пациентов мужчин, пациентов в возрасте младше 60 лет и имеющих среднее образование. Лишь 25,6\% пациентов имели семьи. Подавляющее большинство пациентов $(95,3 \%)$ на момент исследования являлись инвалидами.

В лечении пациентов использовали стандартный режим гемодиализа - 3 раза в неделю продолжительностью от 4 часов. Сосудистый доступ у 78 пациентов обеспечивался артериовенозной фистулой и у 8 - центральным венозным катетером. Продолжительность диализного стажа у пациентов варьировала от 1 месяца до 9 лет и 8 мес. Параметры диализной программы, тактика ведения больных, а также адекватность диализа определяли по действующим клиническим рекомендациям $[11,12]$.

В исследование включали всех больных ХБП 5С, получающих терапию ПГД в стандартном режиме- 3 раза в неделю по 4 ч. Критериями исключения пациентов из исследования были наличие острых воспалительных состояний или обострений хронических заболеваний в течение последнего месяца, наличие злокачественных новообразований, сердечной недостаточности III и IV функционального классов по NYHA, прогрессирующей стенокардии, нарушений ритма сердца и проводимости, состояния гипергидратации на момент обследования и несогласие самого пациента.

Для диагностики депрессии мы использовали одну из самых используемых в исследованиях шкал - шкалу депрессий CES-D (Center for Epidemiological Studies Depression), разработанную в 1977 г. [13]. Опросник содержит 20 вопросов, и дает возможность предположить наличие симптомов депрессии. По результатам суммы баллов шкала предполагает отсутствие симптомов депрессии при наличии менее 18 баллов, легкого депрессивного состояния - 19-26 баллов и выраженного депрессивного состояния - более 26 баллов. Опросник рекомендован для использования терапевтами для предварительной диагностики депрессивной симптоматики.

Для исследования КЖ мы использовали опросник KDQOL-SF ${ }^{\mathrm{T}}$, который был подготовлен в Институте здоровья США, переведен и валидизирован для исполь- 
Таблица 1. Социально-демографическая характеристика пациентов с ХБП С5, получающих лечение ПГД

\begin{tabular}{|l|l|l|l|}
\multicolumn{2}{|l|}{ Параметры } & Абсолютное количество (чел.) & $\begin{array}{l}\text { Относительное количество } \\
\text { (\%) }\end{array}$ \\
\hline \multirow{2}{*}{ Пол } & Мужчины & 60 & 69,8 \\
\cline { 2 - 4 } & Женщины & 26 & 30,2 \\
\hline \multirow{2}{*}{ Возраст (лет) } & До 60 лет & 62 & 72,1 \\
\cline { 2 - 4 } & Старше 60 лет & 24 & 27,9 \\
\hline Наличие семьи & & 22 & 25,6 \\
\hline \multirow{2}{*}{ Образование } & Среднее и средне-специальное & 64 & 74,4 \\
\hline & Высшее & 24 & 25,6 \\
\hline Наличие инвалидности & & 82 & 95,3 \\
\hline
\end{tabular}

Таблица 2. Частота депрессии по шкале CES-D у больных ХБП С5 на ПГД

\begin{tabular}{|c|c|c|c|c|}
\hline \multirow{2}{*}{ Уровень депрессии } & \multicolumn{2}{|c|}{ Мужчины } & \multicolumn{2}{|c|}{ Женщины } \\
\hline & $\mathbf{N}$ & $\%$ & $\mathbf{N}$ & $\%$ \\
\hline Норма & 8 & 13,3 & 2 & 7,6 \\
\hline Легкая депрессия & 32 & 53,4 & 12 & 46,2 \\
\hline Депрессия средней тяжести & 8 & 13,3 & 4 & 15,4 \\
\hline Тяжелая депрессия & 12 & 20 & 8 & 30,8 \\
\hline
\end{tabular}

зования в России [8]. Опросник KDQOL-SF ${ }^{\mathrm{TM}}$ включает в себя 36 пунктов, систематизированных в 8 шкал для оценки физического и психологического здоровья и 43 специфичны для диализных пациентов вопроса. Оценка каждого показателя по опроснику KDQOL-SF ${ }^{\mathrm{TM}}$ производится в баллах от 0 до 100: чем выше балл, тем выше КЖ.

Статистическую обработку результатов исследования мы проводили с использованием программы Statistica 6.0 (Statsoft Inc., USA). Результаты анализа представили как среднее и его стандартное отклонение для непрерывных переменных и как долю (в процентах) для категориальных переменных. За критический уровень значимости при проверке статистических гипотез принимали значения $p<0,05$. Для оценки взаимосвязи качественных признаков применяли метод корреляционного анализа [14].

\section{Результаты и обсужАение}

Частота депрессии по шкале CES-D у обследованных нами больных ХБП С5 на ПГД представлена в табл. 2.

Распространенность депрессии в нашем исследовании оказалась высокой: признаки депрессии среди обследованных нами пациентов не выявлены лишь у 13,3\% мужчин и 7,6\% женщин. Легкая депрессия по шкале CES-D выявлена у 53,4\% мужчин и 46,2\% женщин, депрессия средней тяжести - у 13,3\% мужчин и $15,4 \%$ женщин, а тяжелая депрессия - у $20 \%$ мужчин и $30,8 \%$ женщин.

При проведении корреляционного анализа установлены отрицательные умеренные статистически значимые связи уровня депрессии с большинством доменов КЖ, а именно, с общим состоянием здоровья $(\mathrm{R}=-0,27)$, физическим функционированием $(\mathrm{R}=-0,32)$, интенсивностью боли $(R=-0,29)$, социальным функционированием $(R=-0,29)$, жизненной активностью $(R=-0,46)$, психическим здоровьем $(\mathrm{R}=-0,55)$, физическим компонентом здоровья $(\mathrm{R}=-0,23)$, психологическим компонентом здоровья $(R=-0,51)$, бременем ХБП $(R=-0,48)$, качеством социального взаимодействия $(\mathrm{R}=-0,50)$, когнитивными функциями $(R=-0,36)$, симптомами ХБП $(R=-0,47)$, влиянием ХБП $(R=-0,57)$, сном $(R=-0,27)$.

Сравнительный анализ оценок КЖ обследованных пациентов в зависимости от наличия у них депрессии по шкале CES-D продемонстрировал более высокие показатели по всем доменам у пациентов, набравших не более 17 баллов по шкале CES-D (табл. 3). Различия достигли уровня статистической значимости по доме- 
Таблица 3. Показатели качества жизни пациентов с ХБП С5 на ПГД в зависимости от наличия депрессии (среднее значение \pm стандартное отклонение)

\begin{tabular}{|c|c|c|c|}
\hline Параметры качества жизни (баллы) & $\begin{array}{l}\text { Пациенты, набравшие } 18 \\
\text { и более баллов по шкале } \\
\text { CES-D }\end{array}$ & $\begin{array}{l}\text { Пациенты, набравшие } \\
\text { менее } 18 \text { баллов } \\
\text { по шкале CES-D }\end{array}$ & p \\
\hline Физическое функционирование & $24,7 \pm 22,7$ & $26 \pm 20,9$ & 0,864 \\
\hline Ролевое физическое функционирование & $52,7 \pm 26,7$ & $58,7 \pm 20,5$ & 0,4963 \\
\hline Интенсивность боли & $48,4 \pm 18,9$ & $61 \pm 26,7$ & 0,0630 \\
\hline Общее состояние здоровья & $41,7 \pm 13,1$ & $43,2 \pm 9,4$ & 0,7275 \\
\hline Жизненная активность & $45,1 \pm 18,9$ & $53 \pm 17,5$ & 0,2140 \\
\hline Социальное функционирование & $48,3 \pm 16,5$ & $62,5 \pm 16,7$ & 0,0124 \\
\hline $\begin{array}{l}\text { Ролевое функционирование, обусловленное } \\
\text { эмоциональным состоянием }\end{array}$ & $66,7 \pm 29,8$ & $65,4 \pm 14,5$ & 0,8927 \\
\hline Психическое здоровье & $47,5 \pm 15,2$ & $66,4 \pm 17,5$ & 0,0005 \\
\hline Суммарный физический компонент & $30,7 \pm 6,5$ & $30,5 \pm 9,2$ & 0,9309 \\
\hline Суммарный ментальный компонент & $37,0 \pm 6,2$ & $44,1 \pm 3,9$ & 0,0007 \\
\hline Симптомы и проблемы & $60,9 \pm 15,7$ & $75,8 \pm 19,3$ & 0,0074 \\
\hline Влияние хронической болезни почек & $51,6 \pm 17,4$ & $66,3 \pm 19,3$ & 0,0151 \\
\hline Бремя заболевания почек & $23,4 \pm 17$ & $29,7 \pm 9,9$ & 0,2563 \\
\hline Трудоспособность & $49,3 \pm 6,1$ & 50 & 0,7194 \\
\hline Когнитивные функции & $63,4 \pm 20,5$ & $73,3 \pm 20,4$ & 0,1546 \\
\hline Качество социального взаимодействия & $62,1 \pm 14,9$ & $81,3 \pm 8,2$ & 0,0001 \\
\hline Сексуальные функции & $80,0 \pm 13,2$ & 100 & 0,0000 \\
\hline $\mathrm{CoH}$ & $49,6 \pm 10,8$ & $51 \pm 8,3$ & 0,6945 \\
\hline Общее восприятие здоровья & $48,3 \pm 6,1$ & $54 \pm 19,6$ & 0,0528 \\
\hline Удовлетворенность социальной поддержкой & $54,6 \pm 17,5$ & $63,3 \pm 17,2$ & 0,1425 \\
\hline Поддержка со стороны диализного персонала & $75,7 \pm 15,5$ & $90 \pm 12,9$ & 0,0065 \\
\hline $\begin{array}{l}\text { Удовлетворенность пациента качеством медицинской } \\
\text { помощи }\end{array}$ & $65,4 \pm 16,9$ & $56,7 \pm 8,6$ & 0,1145 \\
\hline
\end{tabular}

нам социального функционирования, психического здоровья, суммарного ментального компонента, симптомов и проблем, влияния ХБП, качества социального взаимодействия, сексуальных функций и поддержки со стороны диализного персонала.

По мнению Kimmel P.L. et al. [4] самым частым психическим расстройством у больных с ХБП С 5 , получающих терапию ПГД, является депрессия. Депрессия у пациентов, получающих заместительную почечную терапию, снижает комплаентность пациентов к лечению [16], увеличивает риск суицида, в т.ч. и пассивных форм последнего в виде отказа от лечения ПГД, а также статистически значимо снижает КЖ пациентов [1].

Таким образом, можно сделать вывод о негативном воздействии симптомов депрессии в виде высоких баллов по опроснику CES-D, на КЖ пациентов с ХБП С5 на ПГД.

\section{Зак^Ючение}

Таким образом, обследованная нами популяция пациентов ХБП С5, получающих терапию ПГД, характеризуется высокой распространенностью депрессии. Установлено, что симптомы депрессии в виде высоких баллов по опроснику CES-D негативно воздействуют на КЖ пациентов с ХБП C5 наПГД. Внедрение использованных нами методик оценки КЖ и уровня депрессии в протокол ведения пациентов с ХБП С5 в диализных центрах позволит мониторировать показатели КЖ и симптомов депрессии пациентов для своевременной коррекции их лечения. Полученные нами результаты можно использовать при планировании психологического и психофармакологического сопровождения пациентов на ПГД. 


\section{ЛИТЕРАТУРА}

1. Kimmel P.L., Cohen S. D., Weisbord S. D. Quality of life in patiens with stage renal disease treated with hemodialysis: survival is not enough // Nephrology. 2008. Vol. 21. Suppl. 13. Pp. 54-59.

2. Бикбов Б.Т., Томилина Н. А. Состав больных и показатели качества лечения на заместительной терапии терминальной хронической почечной недостаточности в Российской Федерации в 1998-2013 гг. (Отчёт по данным российского регистра заместительной почечной терапии. Часть вторая) // Нефрология и диализ. 2016. № 18 (2). С. 98-164.

3. Васильева И. А. Качество жизни больных при лечении гемодиализом: биологические и психосоциальные факторы, методы оценки и подходы к коррекции: автореф. дис. ...д-ра. псих. наук. СПб., 2010. 45 с.

4. Kimmel P.L., Cukor D., Cohen S. D. Depression in end-stage patiens: a critical review // Advances in chronic kidney diseases. 2007. Vol. 14. No. 4. Pp. $328-334$.

5. Bornivelli C., Aperis G., Giannikouris I. et al. Relationship between depression, clinical and biochemical parameters in patients undergoing hemodialysis // J. Ren. Care. 2012. Vol. 38. No. 2. Pp. 93-99.

6. Chilcot J., Wellsted D. Depression in end-stage renaldisease: current advances and re-search // Semin Dial. 2010. Vol. 23. Pp.74-82.

7. Johnstone S. Depression management for hemodialysis patients: Using DOPPS data to further guide nephrology social work intervention // Journal of Nephrology Social Work. 2007. Vol. 26. Pp. 18-31.

8. Васильева И. А. Российская версия опросника Kidney Disease Quality of Life — Short Form (KDQOL-SF $\left.{ }^{\mathrm{m}}\right)$ — ценного диагностического инструмента для оценки качества жизни больных на диализе // Нефрология. 2007. № 11. С. 64-70.

9. Cirillo M., Lombardi C., Chiricone D. et al. Early identification of kidney disease by eGFR: What is the prevalence of eGFR in the population? // J. I Nephrology. 2008. Vol. 21. Suppl. 13. Pp. 102-107.

10. Nobakht E., Cohen S. D. Editorial comment: Health-related quality of life in hemodialysis patients: An Iranian multi-centerstudy // NephroUrology Monthly. 2014. No. 6 (2). Pp.169-186.

11. Строков А.Г., Гуревич К. Я., Ильин А. П., Денисов А. Ю., Земченков А. Ю., Андрусев А. М., Шутов Е. В., Котенко О. Н., Злоказов В. Б. Лечение пациентов с хронической болезнью почек 5 стадии (ХБП 5) методами гемодиализа и гемодиафильтрации. Клинические рекомендации // Программа непрерывного последипломного образования по нефрологии. 2017. № 21 (3). C. 92-111. D0I: 10.24884/1561-6274-2017-3-92-111

12. KDIGO 2012. Clinical Practice Guideline for the Evaluation and Management of Chronic Kidney Disease // Kidney Int Suppl. 2013. Pp. 1-136.

13. Radloff L. S. The CES-D Scale: a self-report depression scale for reseach in the general population // Appl. Psychol. Meas. 1977. No. 1. Pp. 385-401.

14. Реброва 0. Ю. Статистический анализ медицинских данных. Применение пакета прикладных программ Statistica. М.: МедиаСфера, 2002.

15. Jin J., Sklar G., Li S. C. Factors affecting therapeutic compliance: a review from the patient's perspective // Ther Clin Risk Manag. 2008. Vol. 4. Pp. 269-286.

( ) Кардангушева Аксана Мухамедовна ( kardangush@mail.ru ),

Альмова Ирина Хаджиисмаиловна ( almovairina@mail.ru ), Будник Антонина Францевна ( budnik74@mail.ru ), Пшукова Альбина Анатольевна (albina.75.75@bk.ru ), Урусбиева Джаннета Магометовна (j_urusbieva1@mail.ru ), Пшуков Каземир Русланович ( kazik.pshukov@bk.ru).

Журнал «Современная наука: актуальные проблемы теории и практики» 